Bundesgesundheitsbl 2013 · 56:1238-1242

DOI 10.1007/s00103-013-1795-1

Online publiziert: 29. August 2013

(c) Springer-Verlag Berlin Heidelberg 2013

\section{S. Santibanez · A. Mankertz}

Fachgebiet Masern, Mumps, Röteln und Viren bei Abwehrschwäche, Nationales

Referenzzentrum Masern, Mumps, Röteln (NRZ MMR), Robert Koch-Institut, Berlin

\title{
Molekulare Surveillance belegt Fortschritt im Eliminationsprozess der Masern
}

\section{Einleitung}

Masern sind eine hoch ansteckende Krankheit, die mit schwerwiegenden Komplikationen wie Lungen- und Gehirnentzündung einhergehen kann. Die Gefährlichkeit dieser Erkrankung wird vor allem in vielen reichen Ländern unterschätzt: Selbst in Industrieländern ist mit bis zu 3 Todesfällen auf 1000 Masernpatienten zu rechnen. Weltweit sterben jährlich etwa 158.000 Menschen an Masern, diese Todesfälle entfallen vor allem auf Kinder in den Regionen Afrikas und Asiens, die von Mangelernährung und einer hohen HIVInzidenz betroffen sind [1].

Ein sicherer und lang anhaltender Schutz gegen eine Infektion mit dem Masernvirus (MV) wird durch die zweifache Impfung mit dem MMR (oder MMRV)Kombinationsimpfstoff aufgebaut. Für Deutschland hat die Ständige Impfkommission (STIKO) die Impfungen gegen Masern im frühen Kindesalter empfohlen (erste Dosis mit dem vollendeten 11. Lebensmonat, zweite Dosis im Abstand von mindestens 4 Wochen). Darüber hinaus empfiehlt die STIKO allen nach 1970 Geborenen ohne dokumentierten Impfschutz oder mit nur einer Masernimpfung die einmalige Verabreichung des MMR-Impfstoffs [2]. Dieser Impfstoff ist seit seiner Entwicklung vor mehr als 5 Jahrzehnten milliardenfach verabreicht worden. Er ist sicher in der Anwendung und der Schutz vor der Erkrankung hält auch im Erwachsenenalter noch zuverlässig an, Masernerkrankungen werden bei Geimpften nur sehr selten beobachtet.
Das MV infiziert nur den Menschen und eng verwandte Primaten und kann demzufolge durch eine entsprechend hohe Durchimpfung (95\% für beide Impfungen) ausgerottet werden. Die WHO Region Europa strebt die Elimination von Masern und Röteln bis zum Jahr 2015 an [3]. Neben Kriterien wie eine niedrige Inzidenz von Masernfällen bei einer hohen Populationsimmunität werden auch die zeitliche Ausdehnung und die Anzahl der durch eine Transmissionskette ausgelösten Fälle zur Beurteilung der Situation herangezogen. MV können anhand genetischer Merkmale charakterisiert und damit einzelnen Transmissionsketten zugeordnet werden. Die Genotypisierung von MV am Nationalen Referenzzentrum Masern, Mumps, Röteln (NRZ MMR) deckt die Herkunft von importierten MV auf und ermöglicht es, einen untersuchten Fall einem bestimmten Ausbruch zuzuordnen sowie Zusammenhänge zwischen Ausbruchsgeschehen zu erkennen. Die Dynamik des über mehrere Jahre erfassten Muster der MV-Genotypen bildet die Entwicklung der epidemiologischen Situation in einem Land ab; aus der Zirkulationsdauer dominierender MV-Varianten kann auf den erreichten Stand hinsichtlich des Eliminationszieles geschlossen werden. Für die Elimination der Masern muss in der WHO Region Europa dokumentiert werden, dass ein leistungsfähiges Surveillancesystem die über $>12$ Monate anhaltende und damit endemische Transmission einer MVVariante innerhalb der letzten 36 Monate ausschließen kann [3]. Über den Beitrag, den die Genotypisierung von MV für die Elimination der Masern leisten kann, und unsere Erfahrungen für Deutschland berichten wir in diesem Artikel.

\section{Ergebnisse}

Masernviren (MV) können anhand genetischer Merkmale typisiert werden, in dem aus Patientenproben ein $456 \mathrm{Nu}$ kleotide großes Fragment des MV-Genoms, das für den variablen Teil des $\mathrm{Nu}$ kleoproteins N kodiert, durch PCR und Sequenzierung entschlüsselt wird. Die erhaltenen Sequenzdaten werden in einer phylogenetischen Analyse mit den von der WHO festgelegten Referenzstämmen verglichen und in einen Stammbaum eingeordnet. Dieser Stammbaum umfasst 8 mit A bis $\mathrm{H}$ bezeichnete Hauptgruppen (clades), die sich in 24 Genotypen untergliedern, welche durch eine der Hauptgruppe angefügte Zahl gekennzeichnet werden [4].

Dieses standardisierte Verfahren wird weltweit angewendet: Sequenzdaten $\mathrm{zu}$ Masernviren werden fallbezogen mit den zugehörigen epidemiologischen Daten in der WHO-Datenbank MeaNS (Measles Nucleotide Surveillance) archiviert und stehen weltweit für molekular-epidemiologische Analysen zur Verfügung [5]. Damit kann die geografische Verteilung von MV-Genotypen verfolgt werden; so zirkuliert beispielsweise der Genotyp H1 schwerpunktmäßig in China, während der Genotyp B3 charakteristisch für Viren aus der Sub-Sahararegion ist [6]. 


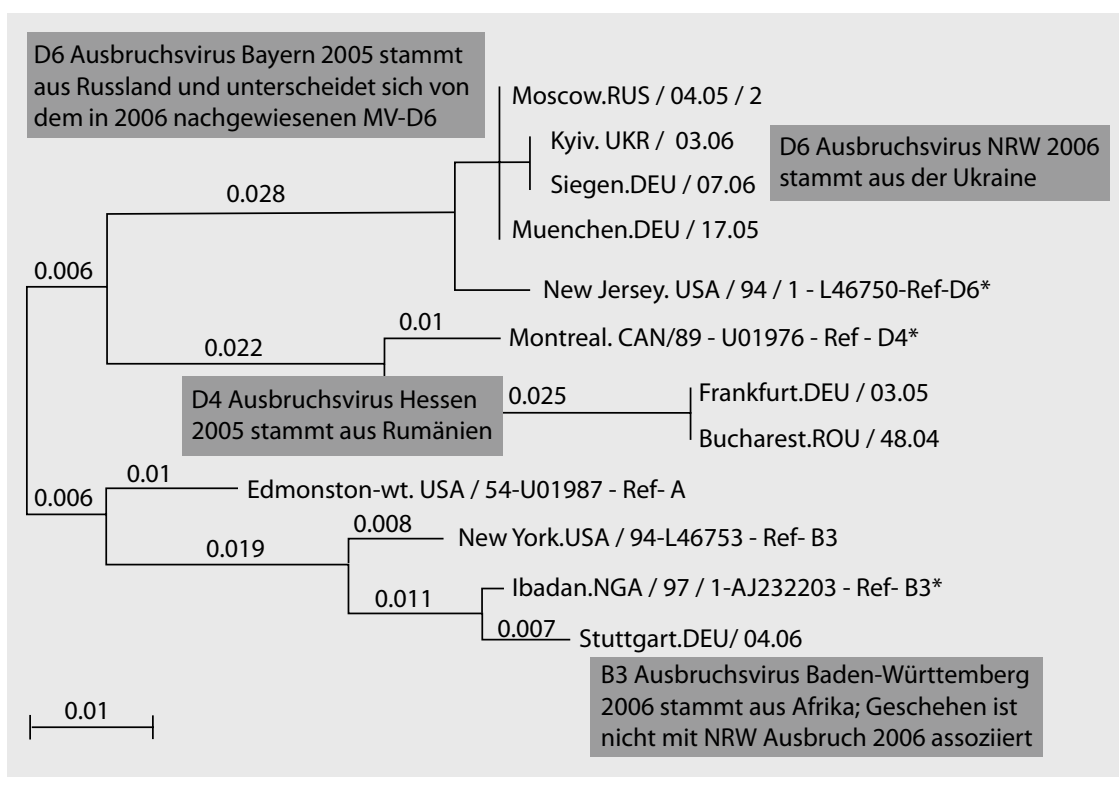

Abb. $1 \Delta$ Die Abbildung zeigt einen Stammbaum von MV der Genotypen B3, D4 und D6, die in den Jahren 2005 und 2006 in Deutschland nachgewiesen wurden. Der Stammbaum basiert auf der Sequenzanalyse eines 456 Nukleotide umfassenden variablen Teils des Nukleoprotein-Gens. Die Zuordnung ist anhand der Einordnung in den entsprechenden Knotenpunkt des Stammbaums bzw. am WHO-Referenzstamm $\left(^{*}\right)$ kenntlich. Die Analyse zeigt, dass die 2005 und 2006 in Bayern und NRW nachgewiesenen D6-MV zu unterschiedlichen Transmissionsketten gehören. Es wird außerdem ersichtlich, dass die gleichzeitig in 2005 beobachteten Ausbrüche in Hessen und Bayern auf verschiedene Genotypen zurückzuführen sind

\section{Einschleppung von Masernviren von und nach Deutschland}

Die Bedeutung der molekularen Surveillance zur Analyse der Herkunft und des Übertragungsweges von MV lässt sich am folgenden Beispiel erläutern: $\mathrm{Zu} \mathrm{Be}$ ginn des Jahres 2010 kam es in Berlin zu einem Masernausbruch mit 62 Fällen [7]. Der Indexfall war ein Schüler einer Berliner Waldorfschule, dessen Familie über Weihnachten eine Reise nach Indien unternommen hatte. Im NRZ MMR wurde der Genotyp D8 festgestellt und das Virus als MVs/Berlin.DEU/03.10 benannt. Das eingeschleppte D8-MV hatte in der Region eine nachweislich über 10 Wochen anhaltende Transmissionskette initiiert. Eine Datenbankabfrage ergab für das Berliner D8-MV Identität mit MVs/Imphal.IND/19.09, das in Indien endemisch zirkuliert. Wir erhielten Material von 13 der 62 Berliner Fälle; in allen wurde der gleiche Genotyp nachgewiesen. Durch die molekulare Surveillance in Kombination mit der epidemiologischen Analyse wurde die Einschleppung dieses D8-MV aus Indien zweifelsfrei bestätigt. D8-MV wurden in Deutschland dern wie Frankreich, das Vereinigte Königreich oder Rumänien beobachtet, wo die Masern noch endemisch sind. Dennoch ist die Masernepidemie in Bulgarien, die 2009 bis 201124.500 Fälle und 24 Todesopfer forderte, auf einen einzigen Masernexport aus Deutschland zurückzuführen [12].

\section{Gleichzeitige Masernausbrüche können verschiedenen Ursprungs sein}

Sowohl in 2005 als auch in 2006 traten zeitlich überlappende Ausbrüche in mehreren Regionen Deutschlands auf. Der Nachweis verschiedener MV-Genotypen zeigte, dass die Geschehen Resultat voneinander unabhängiger MV-Importe waren und somit verschiedenen Transmissionsketten zuzuordnen waren (• Abb. 1).

Von Dezember 2004 bis Mai 2005 ereignete sich in Hessen ein Ausbruch mit rund 220 übermittelten Fällen, der durch die Einschleppung eines D4-MV aus Rumänien ausgelöst worden war. Die dortige Epidemie mit mehr als 8000 gemeldeten Fällen hielt über den Zeitraum 2004 bis 2007 an [13, 14]. Von März bis Juli 2005 trat in Bayern ein Ausbruch mit fast 300 übermittelten Fällen auf [13]. Das hier identifizierte D6-MV war vermutlich aus den Neuen Unabhängigen Staaten eingeschleppt worden, wo die gleiche D6-Variante 2005 noch endemisch zirkulierte.

Eine ähnliche Situation wurde in 2006 beobachtet: $\mathrm{Zu}$ Beginn des Jahres gab es in Baden-Württemberg einen Ausbruch mit mehr als 70 gemeldeten Fällen (Januar bis April), welcher mit einem in Afrika endemischen B3-MV assoziiert war. Zeitgleich hatte sich in NordrheinWestfalen der bisher größte Ausbruch seit dem Abbruch der endemischen Zirkulation entwickelt, welcher annähernd 1700 Fälle (Januar bis Juli) umfasste [15]. Das hier identifizierte D6-MV unterschied sich genetisch von der im Vorjahr in Bayern aufgetretenen Variante, sodass kein Zusammenhang zwischen den Ausbrüchen Bayern 2005 und NordrheinWestfalen 2006 bestanden hat und eine Zirkulation über mehr als $1 \mathrm{Jahr}$ ausgeschlossen werden konnte. Das in Nordrhein-Westfalen identifizierte D6-MV war unmittelbar vor Ausbruchsbeginn 
in Russland und der Ukraine aufgetreten; ein epidemiologischer Zusammenhang mit der Epidemie in der Ukraine mit mehr als 50.000 gemeldeten Fällen von September 2005 bis Juni 2006 [16] kann angenommen werden.

\section{Masern und Massenereignisse}

Masernviren können überall auf Ungeimpfte lauern. Kommen viele Menschen zusammen, werden immer wieder Übertragungsketten in Gang gesetzt. Die Beispiele für MV-Übertragungsketten nach Großereignissen reichen von Sportturnieren in Italien über Rockfestivals in Deutschland bis zu Jugendtreffen in Frankreich. Reisen die infizierten Teilnehmer wieder in ihren jeweiligen Heimatort, kann das Gesamtgeschehen nur bei Zusammenführung der molekularen und epidemiologischen Daten erkannt werden. Im September 2010 traten in Baden-Württemberg und NordrheinWestfalen 6 Masernfälle bei Personen auf, die alle von einem ökumenischen Jugendtreffen im französischen Taizé zurückgekehrt waren. Die Genotypisierung ergab ein D4-MV (Villingen-Schwenningen. DEU/37.10[D4]), das als „D4-Manchester“ (MVs/Manchester.GBR/10.09[D4]) Verursacher großer Ausbrüche in Großbritannien und Frankreich war. Die innerdeutsche Analyse ergab, dass durch die TaizéRückkehrer ein multilokaler Ausbruch mit 13 primären und 17 sekundären Fällen ausgelöst worden war, der die Bundesländer Baden-Württemberg, Nordrhein-Westfalen und Bayern betraf [17].

\section{Verschiedene MV können zu einem Ausbruch beitragen}

Im Jahr 2011 wurde in Baden-Württemberg eine hohe Masernaktivität beobachtet: Von den 525 gemeldeten Fällen traten 194 Erkrankungen im Frühjahr 2011 im Landkreis Ortenaukreis (Regierungsbezirk Freiburg) bei einem Ausbruch an einer Waldorfschule auf [18]. Die Schule wurde geschlossen, um den Ausbruch einzudämmen. Am NRZ MMR wurden Proben von 6 Fällen genotypisiert: Vier Fälle waren bereits kurz vor der Schulschließung erkrankt, während 2 weitere Fälle sich erst danach infiziert hatten. Die zunächst detektierte Masernvirus-Va-

Bundesgesundheitsbl 2013 ·56:1238-1242 DOI 10.1007/s00103-013-1795-1

(c) Springer-Verlag Berlin Heidelberg 2013

\section{S. Santibanez · A. Mankertz \\ Molekulare Surveillance belegt Fortschritt im Eliminationsprozess der Masern}

\section{Zusammenfassung}

Masern werden durch eine Infektion mit dem Masernvirus ausgelöst. Diese schwere Erkrankung führt häufig zu Komplikationen, die in den Industrieländern 1 bis 3 Todesfälle per 1000 Erkrankte verursachen. Masern könnten global ausgerottet werden, wenn mindestens 95\% der Weltbevölkerung zweimalig mit der MMR-Vakzine geimpft wären. Die Elimination der Masern und der Röteln wird für die WHORegion Europa für das Jahr 2015 angestrebt. Ein wichtiges Kriterium für das Gelingen der Elimination ist die Analyse der zeitlichen Dauer der Transmissionsketten, die durch importierte Masernviren ausgelöst werden. Um Masernviren einzelnen Ausbrüchen und Übertragungsketten zuordnen zu können, müs- sen sie genetisch charakterisiert werden. Diese Untersuchungen an Patientenmaterial werden seit dem Start des Interventionsprogrammes für Masern und Röteln im Jahr 1999 kontinuierlich am Nationalen Referenzzentrum Masern, Mumps, Röteln durchgeführt. In diesem Beitrag berichten wir über unsere Erfahrungen und insbesondere über die deutschen Entwicklungen in Bezug auf die Masernelimination.

Schlüisselwörter

Masernvirus · Elimination · Molekulare Surveillance - Genotypisierung · Endemische Zirkulation

\section{Molecular surveillance shows progress in measles elimination process}

\begin{abstract}
Measles is a severe disease caused by infection with the measles virus. Complications after the onset of infection lead to 1-3 fatalities per 1,000 cases in industrialized countries. If more than $95 \%$ of the global population were vaccinated twice with the measles, mumps, and rubella (MMR) vaccine, measles could be eliminated worldwide. The elimination of measles and rubella should be reached in the WHO Europe region in 2015. One important criterion for elimination of the measles virus consists in the analysis of the duration of transmission chains initiated by the import of measles virus. To assign measles viruses to outbreaks and transmis-
\end{abstract}

sion chains, genetic characterization is necessary. These investigations have been performed continually at the National Reference Center Measles, Mumps, Rubella since 1999, when the German Intervention Program was launched. This article summarizes our experiences with measles virus genotyping and new developments with respect to measles elimination in Germany.

\section{Keywords}

Measles virus · Elimination · Molecular surveillance - Genotyping · Endemic circulation riante „D4-Offenburg-1“ unterschied sich im analysierten Genombereich um $2 \mathrm{Nu}$ kleotide von der nachfolgend detektierten Variante „D4-Offenburg-2“. Dieser Befund spricht dafür, dass vor und nach der Schulschließung verschiedene MV-Varianten im Ortenaukreis zirkulierten. Beide Varianten stammen von der in BadenWürttemberg im ersten Halbjahr 2011 dominierenden Variante „D4-Manchester“ ab. Vermutlich hatte die Schulschließung den Abbruch der durch die Variante „D4Offenburg-1“ ausgelösten Infektkette bewirkt; nach den Osterferien wurde wahrscheinlich durch einen weiteren Import eine neue Übertragungskette aufgebaut. Möglicherweise waren auch noch weitere MV-Varianten am Ausbruchsgeschehen beteiligt. Aufgrund der niedrigen Anzahl der zur Typisierung eingesandten Materialien (6 Einsendungen von 194 Fällen) konnte dies aber nicht ermittelt werden. Dieses Beispiel untermalt die dringende Notwendigkeit, in einer Ausbruchssituation eine repräsentative Anzahl von Einsendungen zu untersuchen, um den Erfolg von Interventionsmaßnahmen möglichst objektiv bewerten zu können. 


\section{Abbruch der endemischen Zirkulation in Deutschland}

Das sporadisch erhobene MV-Genotypmuster der 1990er-Jahre zeigte für Deutschland und andere Länder Mittelund Westeuropas (UK, Spanien, Beneluxländer, Tschechische Republik, Polen) eine Dominanz der Genotypen C2 und D6, die deshalb für diese Region als endemisch gelten $[19,20,21,22]$. In den östlichen Bundesländern (BL), die bereits in den 1980erund 1990er-Jahren niedrige Inzidenzen gemeldet hatten, waren im Zeitraum 1999 bis 2002 nur importierte Fälle aufgetreten, die kaum eine Zirkulation ausgelöst hatten; sie waren mit MV der Genotypen B3, D4, D6, D7, G2 und H1 assoziiert [23]. In den westlichen BL war die Masernaktivität deutlich höher; im Zeitraum Oktober 1999 bis November 2003 sind für Bayern, Baden-Württemberg und NordrheinWestfalen die höchsten Inzidenzwerte ermittelt worden. Im Jahr 2000 dominierten in den beiden südlichen BL die damals noch endemisch zirkulierenden $\mathrm{Ge}$ notypen C2 und D6, wohingegen in Nordrhein-Westfalen, Rheinland-Pfalz, Hessen und Schleswig-Holstein bereits der neu aufgetretene Genotyp D7 beobachtet wurde. Für 2001 bis 2002 zeigte das Genotypmuster für die WBL, dass die endemische Zirkulation von C2 und D6 abgebrochen war und stattdessen D7 kontinuierlich zirkulierte [23]. Im Zeitraum 2001 bis 2003 sind C2- und D6-MV durch den neuen Genotyp D7 verdrängt worden, dem es nur in Deutschland gelungen war, eine endemische Zirkulation ( $>3$ Jahre) zu etablieren, die im Jahr 2003 abbrach. Der beobachtete Abbruch der endemischen Zirkulation korreliert mit einem stetigen und deutlichen Rückgang der Maserninzidenz im Zeitraum 2002 bis 2004 von 56,4 auf 1,5.

In den Jahren 2005 bis 2008 kam es zusätzlich zu dem bereits erwähnten großen Ausbruch in Nordrhein-Westfalen in weiteren BL (Hessen, Bayern, Baden-Württemberg) zu Ausbrüchen, die meist mehrere hundert Fälle umfassten, über Monate anhielten, aber nicht auf andere BL übergriffen.

Im Zeitraum 2009-2010 zeichnete sich vor dem Hintergrund von Epidemien in Frankreich ( $>23.000$ Fälle) und Bulgarien (>24.000 Fälle) [24] ein Wandel der epide- miologischen Situation ab. Die in Frankreich dominierende MV-Variante D4Manchester war im Zeitraum 2009 bis 2011 vielfach nach Deutschland eingeschleppt worden; Ausbrüche betrafen überwiegend Baden-Württemberg [17], wo D4-Manchester im Jahr 2011 über 6 Monate (Januar bis Juni) kontinuierlich nachgewiesen wurde. Insgesamt lösten die Importe aber kleinere Geschehen aus, die weniger Fälle und eine kürzere Zirkulationsdauer umfassten. Seit 2009 dominieren in Deutschland die Hauptvarianten D4-Manchester, D4-Hamburg und D4-Enfield. Die z. B. in BadenWürttemberg beobachtete simultane Zirkulation verschiedener MV-Varianten erlaubte die Zuordnung gemeldeter Fälle zu einer bestimmten MV-Variante nur bei erfolgter Typisierung.

Im Jahr 2012 sind bei einer auf 2,0 abgefallenen Inzidenz Daten zum MV-Genotyp für nur 5 Ausbrüche erhoben worden, von denen 3 mit der MV-Variante D4Manchester und 2 mit D4-Marmande assoziiert waren. Eine über mehr als 2 Generationen anhaltende Zirkulation ist jedoch nur für D4-Manchester ermittelt worden; mit 8 Wochen war die Zirkulationsperiode der dominierenden MV-Variante gegenüber den Vorjahren deutlich verkürzt.

\section{Deutschland trägt zur endemischen Zirkulation in Europa bei}

In der Schweiz ist von 2006 bis 2009 eine Epidemie mit mehr als 4400 gemeldeten Fällen abgelaufen, die überwiegend durch ein aus Asien stammendes D5MV (D5-Luzern) verursacht waren [25]. D5-Luzern zirkulierte in der Schweiz kontinuierlich von November 2006 bis März 2009 und bei Sekundärausbrüchen in Deutschland [26, 27], Österreich [28] und Frankreich [29]. Ein weiterer großer Ausbruch ereignete sich infolge einer Einschleppung eines neuen D4MV (Variante D4-Hamburg) aus Großbritannien nach Hamburg im Dezember 2008. Der Ausbruch betraf 269 Fälle in Hamburg und Niedersachsen und hielt bis Juni 2009 an. Die Übertragung dieses Virus nach Bulgarien hatte eine Epidemie mit mehr als 24.500 Fällen zur Folge [12]. Der Ausbruch betraf vor allem die ethnische Gruppe der Roma; es wa- ren 24 Todesfälle zu verzeichnen. Sowohl D5-Luzern als auch D4-Hamburg hatten in Deutschland keine endemische Zirkulation etabliert. Im europäischen Kontext zeigt sich aber eine Verbreitung für 2 bis 3 Jahre. Dies Beispiel belegt eindringlich, dass die Zirkulationsdauer von MV im europäischen Kontext beurteilt werden muss, damit Aussagen für die angestrebte Elimination der Masern in der WHO Region Europa getroffen werden können.

\section{Diskussion}

Um die Elimination der Masern und Röteln in Deutschland zu erreichen, müssten mindestens 95\% der deutschen Bevölkerung zweimalig mit der MMR-Vakzine geimpft sein. Eine ähnlich hohe Durchimpfung hatte in den skandinavischen Ländern einen drastischen Rückgang der Maserninzidenz zur Folge und schützt die Bevölkerung, da Importe keine lang anhaltenden Transmissionsketten initiieren können. Die Genotypisierung von MV ist ein essenzielles Instrument zur Verifizierung der erfolgten MV-Elimination, da die Anzahl der Importe und die Dauer bzw. Fallzahl der ausgelösten Transmissionsketten bestimmt werden können. Aufgrund der Daten ist eine Unterscheidung zwischen endemischer Zirkulation ( $>12$ Monate) und kurzzeitiger Transmission importierter Viren möglich. Der Nachweis des Abbruchs der endemischen Zirkulation ist ein Kernelement für die Verifizierung der Masernelimination.

Die seit dem Jahr 2000 über das Masernsentinel der Arbeitsgemeinschaft Masern (AGM) und bei Einsendungen an das NRZ MMR erhobenen Daten zur Zirkulation genetischer Varianten des MV in Deutschland dokumentieren den Abbruch der Zirkulation der endemischen MV-Genotypen C2, D6 und D7 im Jahr 2003 und zeigen, dass es in der Folgezeit nicht zur erneuten Etablierung einer Zirkulation $>12$ Monate durch ein importiertes MV gekommen ist. Für die Vielzahl der seit 2005 importierten MV wurde eine maximale Zirkulationsperiode von 7 Monaten ermittelt. Die mit einzelnen Ausbrüchen assoziierte Fallzahl und Zirkulationsperiode ist seit 2009 trotz hoher Inzidenz in 2011 rückläufig; im Jahr 2012 fiel bei nur insgesamt 167 in Deutschland gemeldeten Fällen 
die Zirkulationsperiode mit $<2$ Monaten nochmals deutlich kürzer aus. Die nächsten Jahre werden zeigen, ob diese Entwicklung anhält oder ob die Anzahl an NichtGeimpften immer noch zu hoch ist, um die Masernelimination in Deutschland erreichen zu können. Auf europäischer Ebene trägt Deutschland durch Im- und Export von MV zum Erhalt der endemischen MVZirkulation bei.

Der Anteil der Fälle mit ermitteltem MV-Genotyp lag im Zeitraum 2009 bis 2012 zwischen 16 und 21\% der nach IfSG gemeldeten Masernfälle. Eine Untererfassung der Zirkulationsdauer bestimmter MV-Varianten kann deshalb nicht ausgeschlossen werden. Weiterhin war in Regionen mit hoher Inzidenz aufgrund der simultanen Zirkulation verschiedener MV die Zuordnung einzelner Ausbruchsgeschehen zu einer bestimmten Virusvariante erschwert. Für den Zeitraum bis 2011 ist es deshalb fraglich, ob die vorliegenden Genotypisierungsdaten die Viruszirkulation mit ausreichender Zuverlässigkeit abbilden. Mit dem Übergang in die Eliminationsphase ist jedoch zu erwarten, dass die räumliche und zeitliche Dichte der Ausbrüche abnimmt und es somit auch in Deutschland möglich sein wird, für jeden Ausbruch die Virusvariante und deren Zirkulationsdauer zu erfassen um damit ein wesentliches Kriterium für die Verifizierung der Masernelimination erfüllen zu können.

Der Erfolg der MV-Genotypisierung ist stark davon abhängig, ob die zur molekularen Charakterisierung benötigten Proben zur Verfügung stehen. Überregional ist es gleichfalls nötig, dass die Sequenzergebnisse möglichst zeitnah in die entsprechenden weltweit frequentierten Datenbanken eingespeist werden. Um Letzteres werden wir uns bemühen, für Ersteres brauchen wir Ihre Mitwirkung!

Deshalb unsere Bitte an alle niedergelassene Ärzte und Kliniker: Schicken Sie Materialien von jedem Masernfall (Gleiches gilt für Röteln und Mumps) an das Nationale Referenzzentrum zur Genotypisierung. Wir benötigen Zahntaschenflüssigkeit (oral fluid) oder einen Rachenabstrich (feuchter Tupfer) und eine Urinprobe (ca. $2 \mathrm{ml}$ ). Aus Serum kann nicht genotypisiert werden, für manche Fragestellungen kann die zusätzliche Einsendung eines Serums aber hilfreich sein. Entsprechende Entnahmesets (Abnahmematerialien in einem vorfrankierten Versandkarton) können bei uns per E-Mail angefordert werden. Dem Einsender entstehen keine Kosten, der schriftliche Befund geht an den Einsender, die Meldung an das zuständige Gesundheitsamt.

Die Zahl der Masernerkrankungen hat mit bislang 1270 gemeldeten Fällen im Jahr 2013 dramatisch zugenommen (Stand 21.07.2013). Die Erkrankungen treten vor allem im Zusammenhang mit 2 großen Ausbrüchen in Berlin (460 Fälle) und Bayern (540 Fälle) auf. Seit Mitte Februar wird fast ausnahmslos die MV-Variante „D8Frankfurt am Main“ nachgewiesen. Durch die fortgesetzte molekulare Surveillance wird erkennbar, dass die positive Entwicklung im Jahr 2012 keine Trendwende eingeläutet hat, sondern importierte MV weiterhin lang anhaltende Transmissionsgeschehen auslösen und Deutschland das Eliminationsziel für die Masern verfehlen wird.

\section{Korrespondenzadresse}

\section{Dr. S. Santibanez}

Fachgebiet Masern, Mumps, Röteln und Viren bei Abwehrschwäche, Nationales Referenzzentrum Masern, Mumps, Röteln (NRZ MMR), Robert Koch-Institut

Nordufer 20, 13353 Berlin

santibanezs@rki.de

Danksagung. S. Santibanez und A. Mankertz danken allen Ärzten und Gesundheitsämtern, die Proben an das NRZ MMR schicken, und Ihren Kolleginnen Ingrid Deitemeier, Petra Kurzendörfer, Cornelia Lentz, Christine Schwerdtfeger und Anne Wolbert für die engagierte technische Assistenz.

Interessenkonflikt. S. Santibanez und A. Mankertz geben an, dass kein Interessenkonflikt besteht.

Dieser Beitrag beinhaltet keine Studien an Menschen oder Tieren

\section{Literatur}

1. WHO Media Centre: Measles (2013) Fact sheet $\mathrm{N}^{\circ} 286$ : http://www.who.int/mediacentre/factsheets/fs286/en/

2. Robert Koch-Institut (RKI) (2012) Empfehlungen der Ständigen Impfkommission (STIKO) am Robert KochInstitut. Epid Bull Nr. 30/2012

3. WHO Regional Office for Europe (2012) Eliminating measles and rubella. Framework for the verification process in the WHO European Region. http://www. euro.who.int/_data/assets/pdf_file/0005/156776/ e96153-Eng-final-version.pdf

4. WHO (2012) Measles virus nomenclature update: 2012. Weekly epidemiological record No. 9, 2012. 87:73-80. http://www.who.int/wer

5. Gnaneshan S, Brown KE, Green J, Brown DW (2008) Online global/WHO-European regional measles nucleotide surveillance. Euro Surveill 13:19
6. Rota PA, Brown K, Mankertz A et al (2011) Global distribution of measles genotypes and measles molecular epidemiology. J Infect Dis 204(Suppl 1):S514-S523

7. Bätzing-Feigenbaum J, Pruckner U, Beyer A et al (2010) Spotlight on measles 2010: preliminary report of an ongoing measles outbreak in a subpopulation with low vaccination coverage in Berlin, Germany, JanuaryMarch 2010. Euro Surveill 15:13

8. Robert Koch-Institut (RKI) (2007) Infektionsgeschehen von besonderer Bedeutung, Masern: Zu den aktuellen Ausbruchsgeschehen in Nordrhein-Westfalen und Niederbayern. Epid Bull Nr. 17/2007

9. Roggendorf $H$, Mankertz $A$, Kundt R, Roggendorf $M$ (2010) Spotlight on measles 2010: measles outbreak in a mainly unvaccinated community in Essen, Germany, March-June 2010. Euro Surveill 15(26)

10. Oster NV, Harpaz R, Redd SB, Papania MJ (2004) International importation of measles virus - United States, 1993-2001. J Infect Dis 189:S48-S53

11. Centers for Disease Control and Prevention (2011) Measles - United States, 2011. MMWR Morb Mortal Wkly Rep 61:253-257

12. Mankertz A, Mihneva Z, Gold H et al (2011) Spread of measles virus D4-Hamburg in Europe, 2008-2011. Emerg Infect Dis 17(8):1396-1401

13. Siedler A, Tischer A, Mankertz A, Santibanez S (2006) Two outbreaks of measles in Germany 2005. Euro Surveill 11(4)

14. Kremer JR, Brown KE, Jin L et al (2008) High genetic diversity of measles virus, World Health Organization European Region, 2005-2006. Emerg Infect Dis 14:107-114

15. Wichmann O, Siedler A etal (2009) Further efforts needed to achieve measles elimination in Germany: results of an outbreak investigation. Bull World Health Organ 87:108

16. Velicko I, Müller LL, Pebody R et al (2008) Nationwide measles epidemic in Ukraine: the effect of low vaccine effectiveness. Vaccine 26(52):6980-6985

17. Pfaff G, Lohr D, Santibanez S et al (2010) Spotlight on measles 2010: measles outbreak among travellers returning from a mass gathering, Germany, SeptemberOctober 2010. Euro Surveill 15(50)

18. Robert Koch-Institut (RKI) (2012) Auf dem Weg zur Elimination der Masern in Deutschland: Aktuelle Epidemiologie und Erfahrungen aus Ausbruchsuntersuchungen 2010/2011. Epid Bull Nr. 19/2012

19. Jin L, Brown DWG, Ramsay ME et al (1997) The diversity of measles in the United Kingdom, 1992-1995. J Gen Virol 78:1287-1294

20. Rima BK, Earle JAP, Yeo RP et al (1995) Temporal and geographical distribution of measles virus genotypes. J Gen Virol 76:1173-1180

21. Hanses F, Binnendijk R van, Ammerlaan W et al (2000) Genetic variability of measles virus circulating in the Benelux. Arch Virol 145:541-551

22. Santibanez S, Heider A, Gerike E et al (1999) Genotyping of measles virus isolates from Central Europe and Russia. J Med Virol 58:313-320

23. Santibanez S, Tischer A, Heider A et al (2002) Rapid replacement of endemic measles virus genotypes. J Gen Virol 83:2699-2708

24. WHO Regional Office for Europe (2013) Centralized information system for infectious diseases (CISID). http:// data.euro.who.int/cisid/?TabID=304266

25. Richard JL, Masserey-Spicher V, Santibanez S, Mankertz A (2008) Measles outbreak in Switzerland - an update relevant for the European football championship (EURO 2008). Eurosurveillance 13:8

26. Bernard H, Santibanez S, Siedler A et al (2007) An outbreak of measles in Lower Bavaria, Germany, January-June 2007. Euro Surveill 12:E071004.1

27. Pfaff G, Mezger B, Santibanez S et al (2008) Measles in south-west Germany imported from Switzerland a preliminary outbreak description. Euro Surveill 13:8

28. Schmid D, Holzmann H, Schwarz K et al (2009) Measles outbreak linked to a minority group in Austria, 2008. Epidemiol Infect 14:1-11

29. Waku-Kouomou D, Freymuth F, Chatelet IP du et al (2010) Co-circulation of multiple measles virus genotypes during an epidemic in France in 2008. J Med Virol 82:1033-1043 